\title{
Prognostic significance of L1CAM expression and its association with mutant p53 expression in high-risk endometrial cancer
}

Inge C Van Gool ${ }^{1}$, Ellen Stelloo ${ }^{1}$, Remi A Nout ${ }^{2}$, Hans W Nijman ${ }^{3}$, Richard J Edmondson ${ }^{4}$, David N Church ${ }^{5,6}$, Helen J MacKay ${ }^{7}$, Alexandra Leary ${ }^{8}$, Melanie E Powell9, Linda Mileshkin ${ }^{10}$, Carien L Creutzberg ${ }^{2}$, Vincent THBM Smit ${ }^{1}$ and Tjalling Bosse ${ }^{1}$

${ }^{1}$ Department of Pathology, Leiden University Medical Center, Leiden, The Netherlands; ${ }^{2}$ Department of Radiation Oncology, Leiden University Medical Center, Leiden, The Netherlands; ${ }^{3}$ Department of Gynecology, University Medical Center Groningen, Groningen, The Netherlands; ${ }^{4}$ Institute of Cancer Sciences, University of Manchester, St Marys Hospital, Manchester, UK; ${ }^{5}$ Molecular and Population Genetics Laboratory, The Wellcome Trust Centre for Human Genetics, University of Oxford, Oxford, UK; ${ }^{6}$ Oxford Cancer Centre, Churchill Hospital, Oxford, UK; ${ }^{7}$ Division of Gynecologic Oncology, Department of Obstetrics and Gynecology, Princess Margaret Hospital/University Health Network, University of Toronto, Toronto, Ontario, Canada; ${ }^{8}$ Department of Medicine, Gynecology Unit, Gustave Roussy, Villejuif, France; ${ }^{9}$ Department of Clinical Oncology, Barts Health NHS Trust, London, UK and ${ }^{10}$ Division of Cancer Medicine, Peter MacCallum Cancer Centre, East Melbourne, VIC, Australia

Studies in early-stage, predominantly low- and intermediate-risk endometrial cancer have demonstrated that L1 cell adhesion molecule (L1CAM) overexpression identifies patients at increased risk of recurrence, yet its prognostic significance in high-risk endometrial cancer is unclear. To evaluate this, its frequency, and the relationship of L1CAM with the established endometrial cancer biomarker p53, we analyzed the expression of both markers by immunohistochemistry in a pilot series of 116 endometrial cancers (86 endometrioid, 30 non-endometrioid subtype) with high-risk features (such as high tumor grade and deep myometrial invasion) and correlated results with clinical outcome. We used The Cancer Genome Atlas (TCGA) endometrial cancer series to validate our findings. Using the previously reported cutoff of $10 \%$ positive staining, $51 / 116(44 \%)$ tumors were classified as L1CAM-positive, with no significant association between L1CAM positivity and the rate of distant metastasis $(P=0.195)$. However, increasing the threshold for L1CAM positivity to $50 \%$ resulted in a reduction of the frequency of L1CAM-positive tumors to $24 \%(28 / 116)$, and a significant association with the rate of distant metastasis $(P=0.018)$. L1CAM expression was strongly associated with mutant p53 in the high-risk and TCGA series $(P<0.001)$, although a substantial fraction $(36 \%$ of endometrioid, $10 \%$ of non-endometrioid morphology) of p53-mutant endometrial cancers displayed $<10 \%$ L1CAM positivity. Moreover, $30 \%$ of p53-wild-type non-endometrioid endometrial cancers demonstrated diffuse L1CAM staining, suggesting p53-independent mechanisms of L1CAM overexpression. In conclusion, the previously proposed threshold for L1CAM positivity of $>10 \%$ does not predict prognosis in high-risk endometrial cancer, whereas an alternative threshold $(>50 \%)$ does. L1CAM expression is strongly, but not universally, associated with mutant p53, and may be strong enough for clinical implementation as prognostic marker in combination with p53. The high frequency of L1CAM expression in high-risk endometrial cancers suggests that it may also be a promising therapeutic target in this tumor subset.

Modern Pathology (2016) 29, 174-181; doi:10.1038/modpathol.2015.147; published online 8 January 2016

Correspondence: Dr T Bosse, MD, PhD, Department of Pathology, Leiden University Medical Center, PO Box 9600, Leiden 2300 RC, The Netherlands.

E-mail: T.Bosse@lumc.nl

Received 2 September 2015; accepted 12 November 2015; published online 8 January 2016
A major challenge in the treatment of endometrial cancer is the identification of patients likely to benefit from adjuvant therapy from those that do not require additional treatment after surgery. Currently, the clinicopathologic criteria, including (combinations of) older age, advanced disease stage, 
high tumor grade, non-endometrioid histology, and substantial lymphovascular space invasion, are used to identify the $\sim 15-20 \%$ of patients at high risk of recurrence for whom postoperative external beam radiation therapy and/or chemotherapy are usually recommended. Over- and undertreatment, however, remain a clinical problem. Consequently, much attention has focused on the identification of prognostic biomarkers that improve current clinicopathologic risk stratification. Recent studies have identified POLE proofreading mutation, TP53 mutation, and microsatellite instability status as promising endometrial cancer biomarkers, ${ }^{1-5}$ yet there remains a pressing need to validate other novel prognostic and predictive markers for clinical use.

One such promising candidate is L1 cell adhesion molecule (L1CAM; CD171), a membrane glycoprotein of the immunoglobulin superfamily that has an important role in neurogenesis by regulating cell adhesion and migration. ${ }^{6-8}$ In endometrial cancer and other malignancies, L1CAM expression in tumor cells promotes disease progression by augmentation of cell motility, invasion, and metastasis. ${ }^{6,9-17}$ In two large recent series of early-stage, predominantly low- and intermediate-risk endometrioid endometrial cancers, L1CAM positivity was observed in $7-18 \%$ of tumors, where it was shown to identify patients at high risk of disease recurrence within an $a$ priori low-risk group (HR 2.05-15.01, $P<0.001$ in both studies). ${ }^{18,19}$ The threshold for L1CAM positivity used in these studies $(>10 \%$ of tumor cells with L1CAM staining) was initially selected based on the greatest discrimination between L1CAM-positive and -negative cancer with respect to prognosis and was validated in an independent series. ${ }^{18,19}$ Patients with (high) L1CAM expression may benefit from more intensive adjuvant treatment or potentially from antibody-mediated therapy against L1CAM itself. However, the frequency and prognostic significance, as well as the optimal threshold of L1CAM expression in high-risk endometrial cancer, remain to be elucidated.

In the previous studies, non-endometrioid endometrial cancers, frequently carrying TP53 mutations, or even foci of non-endometrioid differentiation in an otherwise endometrioid endometrial cancer were significantly more often L1CAM-positive compared with tumors with pure endometrioid histology $\left(P=0.003\right.$ and $<0.001$, respectively). ${ }^{18,19}$ Because of this strong association, both reports addressed the possibility of L1CAM positivity in endometrioid endometrial cancer representing missed cell clusters of non-endometrioid differentiation. Given the mechanistic association between L1CAM and p53, ${ }^{20}$ as well as the association of TP53 mutations with poor prognosis in early-stage endometrial cancer, ${ }^{2,3}$ understanding whether L1CAM is an independent prognostic biomarker of merely a surrogate for TP53 mutation is essential to determine its clinical utility.
Our objectives therefore were to determine the frequency and prognostic significance of L1CAM expression and its relationship with p53 in an international pilot series of high-risk endometrial cancers. $^{3}$

\section{Materials and methods}

\section{Patient and Tissue Selection}

High-risk endometrial cancer samples were collected from collaborating institutions within the TransPORTEC consortium. The inclusion criteria for high-risk patients (as handled in PORTEC-3) included endometrioid endometrial cancer with one of the following postoperative FIGO 2009 stages and grade: stage IA grade 3 with myometrial invasion, stage IB grade 3 or higher stages, and serous or clear-cell histology with stage IA with myometrial invasion, or higher stages. Patient $(N=116)$ and tissue selection have been described previously, ${ }^{3}$ and characteristics are shown in Table 1.

\section{Immunohistochemical Staining and Evaluation}

Immunohistochemistry was performed as described earlier. ${ }^{18,21}$ Briefly, the formalin-fixed, paraffinembedded tissue blocks were cut into $4 \mu \mathrm{m}$ slides and mounted on Starfrost slides. Endogenous peroxidases were inactivated by $0.3 \% \mathrm{H}_{2} \mathrm{O}_{2}$ /methanol. Antigen retrieval was achieved by microwave oven treatment in $10 \mathrm{mmol} / \mathrm{l}$ Tris-EDTA, $\mathrm{pH}$ 9.0. Sections were incubated overnight with primary monoclonal antibodies against L1CAM (CD171; clone 14.10; 1:500; SIG-3911; Convance Inc.) or against p53 (clone DO-7; 1:2000; Neomarkers; Ab-5). The sections were incubated for $30 \mathrm{~min}$ with a secondary antibody (poly-HRP-GAM/R/R; DPV0110HRP; ImmunoLogic). Diaminobenzidine tetrahydrochloride was used as a chromogen. The slides were counterstained with Mayer's haematoxylin, dehydrated and mounted. As an external positive control, a highly L1CAM-expressing serous ovarian carcinoma was used.

Immunohistochemical evaluation was independently conducted by two investigators blinded for patient characteristics and outcome, with discrepancies resolved by consensus under a multihead microscope. The percentage of positive membranous L1CAM staining within the tumor was scored as $0 \%$, $<10 \%, 10-50 \%$, or $>50 \%$, according to a recently published scoring system for endometrial cancer. ${ }^{19}$ If the tumor stained $>10 \%$, it was considered L1CAMpositive, based on prior studies. ${ }^{18,19}$ Nerves running through the deeper myometrium served as internal positive controls and were identified in all cases. P53 was scored mutant if $>50 \%$ of the tumor cells of the tumor as a whole or if $>50 \%$ of the tumor cells of a discrete geographical area showed strong positive nuclear staining. ${ }^{22}$ 
Table 1 Clinicopathologic patient characteristics in relation to L1CAM expression $(N=116)$

\begin{tabular}{|c|c|c|c|c|c|c|c|}
\hline & \multicolumn{2}{|c|}{ Total } & \multicolumn{2}{|c|}{$L 1 C A M<10 \%$} & \multicolumn{2}{|c|}{$L 1 C A M \geq 10 \%$} & \multirow[t]{2}{*}{$\mathrm{P}$-value } \\
\hline & $(N=116)$ & $\%$ & $(N=65)$ & $\%$ & $(N=51)$ & $\%$ & \\
\hline \multicolumn{8}{|l|}{ Age } \\
\hline Mean (range) & 66.3 & $21-85$ & 64.3 & $45-82$ & 68.7 & $21-85$ & 0.033 \\
\hline$\leq 60$ & 38 & 32.8 & 26 & 40.0 & 12 & 23.5 & 0.045 \\
\hline $61-70$ & 31 & 26.7 & 19 & 29.2 & 12 & 23.5 & \\
\hline$>70$ & 47 & 40.5 & 20 & 30.8 & 27 & 53.0 & \\
\hline \multicolumn{8}{|l|}{ FIGO stage 2009} \\
\hline I & 42 & 36.2 & 24 & 36.9 & 18 & 35.3 & 0.488 \\
\hline II & 21 & 18.1 & 13 & 20.0 & 8 & 15.7 & \\
\hline III & 41 & 35.3 & 20 & 30.8 & 21 & 41.1 & \\
\hline IV & 11 & 9.5 & 8 & 12.3 & 3 & 5.9 & \\
\hline Unknown & 1 & 0.9 & 0 & 0.0 & 1 & 2.0 & \\
\hline \multicolumn{8}{|l|}{ Tumor type } \\
\hline Endometrioid & 86 & 74.1 & 58 & 89.2 & 28 & 54.9 & $<0.001$ \\
\hline Serous & 12 & 10.4 & 2 & 3.1 & 10 & 19.6 & \\
\hline Clear cell & 18 & 15.5 & 5 & 7.7 & 13 & 25.5 & \\
\hline \multicolumn{8}{|l|}{ Grade } \\
\hline 1 & 13 & 11.2 & 12 & 18.4 & 1 & 2.0 & $0.022^{\mathrm{a}}$ \\
\hline 2 & 5 & 4.3 & 5 & 7.7 & 0 & 0.0 & $<0.001^{\mathrm{b}}$ \\
\hline 3-Endometrioid & 68 & 58.6 & 41 & 63.1 & 27 & 52.9 & \\
\hline 3-Non-endometrioid & 30 & 25.9 & 7 & 10.8 & 23 & 45.1 & \\
\hline \multicolumn{8}{|c|}{ Depth of myometrial invasion } \\
\hline$<50 \%$ & 23 & 19.8 & 13 & 20.0 & 10 & 19.6 & 0.856 \\
\hline$>50 \%$ & 87 & 75.0 & 51 & 78.5 & 36 & 70.6 & \\
\hline Unknown & 6 & 5.2 & 1 & 1.5 & 5 & 9.8 & \\
\hline \multicolumn{8}{|c|}{ Lymphovascular space invasion } \\
\hline Absent & 40 & 34.5 & 25 & 38.4 & 15 & 29.4 & 0.946 \\
\hline Present & 55 & 47.4 & 34 & 52.3 & 21 & 41.2 & \\
\hline Unknown & 21 & 18.1 & 6 & 9.3 & 15 & 29.4 & \\
\hline \multicolumn{8}{|l|}{ Adjuvant radiotherapy } \\
\hline EBRT & 55 & 47.4 & 37 & 56.9 & 18 & 35.3 & 0.473 \\
\hline VBT & 1 & 0.9 & 0 & 0.0 & 1 & 2.0 & \\
\hline $\mathrm{EBRT}+\mathrm{VBT}$ & 21 & 18.1 & 12 & 18.5 & 9 & 17.6 & \\
\hline None & 15 & 12.9 & 10 & 15.4 & 5 & 9.8 & \\
\hline Unknown & 24 & 20.7 & 6 & 9.2 & 18 & 35.3 & \\
\hline \multicolumn{8}{|l|}{ Adjuvant chemotherapy } \\
\hline Yes & 16 & 13.8 & 9 & 76.9 & 7 & 13.7 & 0.470 \\
\hline No & 76 & 65.5 & 50 & 13.9 & 26 & 51.0 & \\
\hline Unknown & 24 & 20.7 & 6 & 9.2 & 18 & 35.3 & \\
\hline
\end{tabular}

Abbreviations: EBRT, external beam radiotherapy; L1CAM, L1 cell adhesion molecule; VBT; vaginal brachytherapy.

${ }^{a} P$-value calculated using endometrioid-type tumors only (grades $1-3, N=86$ ).

${ }^{\mathrm{b}} P$-value calculated over the complete cohort $(N=116)$.

\section{TP53 Mutation Analysis}

Tumor DNA was isolated, and Sanger sequencing for exons 5-8 of TP53 was performed on the samples that showed an indefinite p53 immunohistochemical staining pattern as described previously. ${ }^{1,3}$ Compared with the earlier study on the same cohort, ${ }^{3}$ careful revision of the p53 immunohistochemical staining resulted in two additional cases being scored as p53-indefinite instead of p53-wild-type because of errors in fixation; Sanger sequencing revealed p.R283H and p.G245S mutations in these two respective cases.

\section{TCGA Endometrial Cancer Series}

We used publicly available endometrial cancer data from The Cancer Genome Atlas (TCGA) series for validation of our results. ${ }^{2}$ RSEM-normalized RNA-Seq data were downloaded from FireBrowse (http://firebrowse.org/?cohort = UCEC\& download_dialog = true, accessed 11 December 2014) and annotated according to available clinicopathogic data and TP53 mutation status. ${ }^{23}$ Following removal of normal tissue controls and technical duplicates, 245 samples were informative for analysis. 

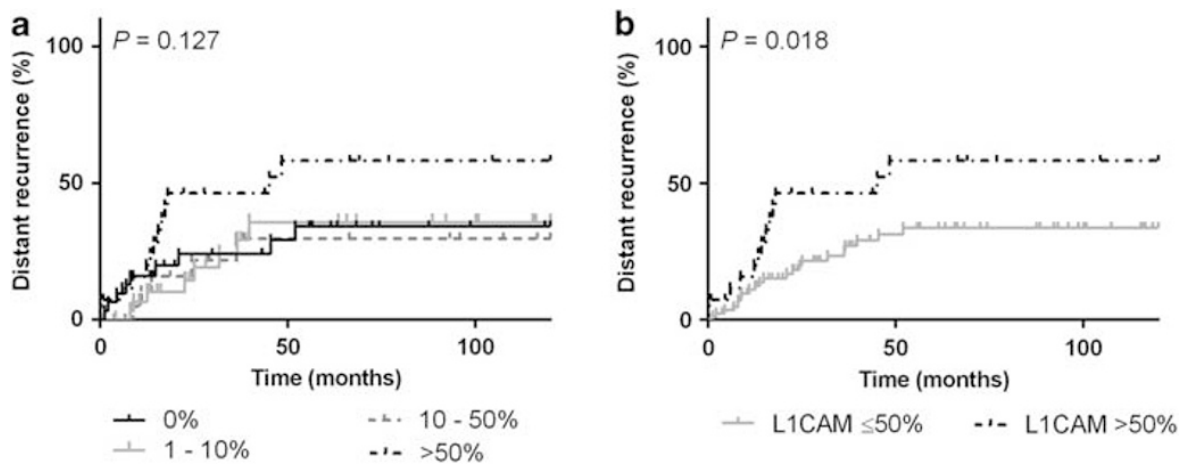

Figure 1 Rate of distant recurrences in high-risk endometrial cancer patients stratified by L1 cell adhesion molecule (L1CAM) expression as determined by immunohistochemistry. L1CAM expression was scored into four groups based on the percentage of positive membranous staining (a) and into two groups with positivity defined as membranous staining in $>50 \%$ of tumor cells (b).

\section{Statistical Analyses}

Statistical analyses were performed using the IBM SPSS for Windows 20.0 software. Patient and tumor characteristics were compared with $t$-test for continuous variables and with $\chi^{2}$ statistics or Fisher's exact test for categorical variables. Rates of distant recurrences, locoregional recurrences, recurrencefree, and overall survival were analyzed using Kaplan-Meier method and log-rank test starting at the date of diagnosis, with censoring of patients alive and free of recurrence at the date of last follow-up. For analysis of overall survival, all deaths irrespective of cause were considered an event; for recurrence-free survival, all recurrences (locoregional and distant) were considered an event. For the TCGA series, the optimal threshold for L1CAM positivity was calculated by unpruned classification and regression decision tree with a 10 -fold crossvalidation. ${ }^{19}$ Using disease-free survival status as dependent variable, this decision tree resulted in a binary scoring method with L1CAM positivity defined as an expression $>132.12$ (data not shown). All statistical tests were two-sided and $P$-values $<0.05$ were considered statistically significant.

\section{Results}

Clinicopathologic characteristics of the 116 included high-risk samples and their relationship with L1CAM expression are shown in Table 1. Fifty-one $(44 \%)$ tumors were found to be L1CAM-positive (cutoff value 10\%). L1CAM positivity was significantly associated with advanced age (68.7 vs 64.3 years, $P=0.033$ ), non-endometrioid morphology $(P<0.001)$, and high tumor grade $(P<0.001)$. Subanalysis in endometrioid endometrial cancers also revealed a significant association between L1CAM expression and high tumor grade $(P=0.022)$. No significant association was found between L1CAM expression and FIGO stage, depth of myometrial invasion, and/or the presence of lymphovascular space invasion.

In univariate analysis, L1CAM-positive tumors did not have a significantly different rate of distant recurrences $(P=0.195)$. Median follow-up time was 28.6 months (range 0.3-120 months). Distant metastasis rate for tumors with L1CAM expression in the lowest three categories $(0,<10$, and $10-50 \%)$ were similar, whereas, in comparison, the rate in case of $>50 \%$ L1CAM expression was higher (Figure 1a). Using this higher threshold, $24 \%(28 / 116)$ of all tumors were L1CAM-positive (Supplementary Table 1 ), and the rate of distant recurrences was significantly higher in L1CAM-positive tumors $(P=0.018$; Figure 1b). Data on overall survival and recurrences are summarized in Table 2.

Next, we examined the relationship between L1CAM and mutant p53 expression (Table 3). A significant association was found with p53-mutant tumors often showing diffuse L1CAM expression $(P=0.001)$. Of p53-mutant tumors with non-endometrioid morphology, $75 \%$ were strongly L1CAM-positive $(>50 \% ; \quad P=0.031)$, whereas in tumors with endometrioid morphology, a nonsignificant yet similar distribution was seen $(P=0.471)$. Despite the strong association, $10 \%$ of p53-mutant non-endometrioid tumors display $<10 \%$ L1CAM expression, whereas also $30 \%$ of p53-wild-type non-endometrioid tumors are diffusely L1CAMpositive $(>50 \%)$. For tumors with endometrioid morphology, the proportion of $<10 \%$ L1CAM expression in p53-mutant tumors is higher (59\%), with $36 \%$ being completely L1CAM-negative. Examples of these results are shown in Figure 2. L1CAM (cutoff of 50\%) and p53 expression combined in univariate analysis for rate of distant recurrences suggests added prognostic value of L1CAM to p53 as the highest rate was seen in combined mutant p53 and $>50 \%$ L1CAM expression $(P=0.031$; Supplementary Figure 1).

We sought to validate our findings regarding the association of p53 and L1CAM, using the publicly available TCGA endometrial cancer series, 
Table 2 Clinical outcomes by L1CAM expression, by p53 expression, and by combined diffuse L1CAM expression and mutant p53 expression

\begin{tabular}{|c|c|c|c|c|c|c|c|c|c|c|c|c|c|c|}
\hline & \multicolumn{2}{|c|}{$\begin{array}{l}\text { L1CAM } 0 \% \\
(\mathrm{~N}=31)\end{array}$} & \multicolumn{2}{|c|}{$\begin{array}{l}\text { L1CAM }<10 \% \\
\quad(\mathrm{~N}=34)\end{array}$} & \multicolumn{2}{|c|}{$\begin{array}{l}\text { L1CAM 10-50\% } \\
(\mathrm{N}=23)\end{array}$} & \multicolumn{2}{|c|}{$\begin{array}{c}\text { L1CAM }>50 \% \\
\quad(\mathrm{~N}=27)\end{array}$} & \multicolumn{2}{|c|}{$\begin{array}{l}\text { P53-wild-type } \\
\qquad(\mathrm{N}=74)\end{array}$} & \multicolumn{2}{|c|}{$\begin{array}{l}\text { P53-mutant } \\
(\mathrm{N}=41)\end{array}$} & \multicolumn{2}{|c|}{$\begin{array}{l}\text { L1CAM }>50 \% \text { p53- } \\
\text { mutant }(\mathrm{N}=18)\end{array}$} \\
\hline & Events & $\%$ & Events & $\%$ & Events & $\%$ & Events & $\%$ & Events & $\%$ & Events & $\%$ & Events & $\%$ \\
\hline \multicolumn{15}{|l|}{ Survival } \\
\hline Alive & 22 & 71.0 & 22 & 64.7 & 12 & 52.2 & 11 & 40.7 & 49 & 66.2 & 18 & 43.9 & 8 & 44.4 \\
\hline Dead & 9 & 29.0 & 12 & 35.3 & 11 & 47.8 & 16 & 59.3 & 25 & 33.8 & 23 & 56.1 & 10 & 55.6 \\
\hline \multicolumn{15}{|l|}{ Recurrence } \\
\hline Overall & 11 & 35.5 & 12 & 35.3 & 7 & 30.4 & 14 & 50.0 & 23 & 31.3 & 21 & 50.0 & 12 & 63.2 \\
\hline Locoregional & 3 & 9.7 & 4 & 12.1 & 2 & 9.5 & 5 & 18.5 & 8 & 10.8 & 6 & 15.8 & 4 & 22.2 \\
\hline Distant & 9 & 29.0 & 8 & 24.2 & 5 & 22.7 & 13 & 48.1 & 17 & 23.0 & 18 & 46.2 & 11 & 61.1 \\
\hline
\end{tabular}

Abbreviation: L1CAM, L1 cell adhesion molecule.

Table 3 Relationship between L1CAM and p53 expression in high-risk endometrial cancer grouped by histology

\begin{tabular}{|c|c|c|c|c|c|}
\hline & \multicolumn{5}{|c|}{ L1CAM expression } \\
\hline & $0 \%$ & $<10 \%$ & $10-50 \%$ & $>50 \%$ & Total (\%) \\
\hline \multicolumn{6}{|l|}{ Total $(P=0.001)$} \\
\hline p53-wild-type & $23(31.1)$ & $27(36.5)$ & $15(20.3)$ & $9(12.1)$ & 74 \\
\hline p53-mutant & $8(19.0)$ & 7 (16.7) & $8(19.0)$ & $19(45.2)$ & 42 \\
\hline Total & $31(26.7)$ & $34(29.3)$ & $23(19.8)$ & $28(24.2)$ & 116 \\
\hline \multicolumn{6}{|c|}{ Endometrioid $(P=0.471)$} \\
\hline p53-wild-type & $20(31.2)$ & 25 (39.1) & $13(20.3)$ & $6(9.4)$ & 64 \\
\hline p53-mutant & $8(36.4)$ & $5(22.7)$ & $5(22.7)$ & $4(18.2)$ & 22 \\
\hline Total & $28(32.6)$ & $30(34.9)$ & $18(20.9)$ & $10(11.6)$ & 86 \\
\hline \multicolumn{6}{|c|}{ Non-endometrioid ${ }^{\mathrm{a}}(P=0.031)$} \\
\hline p53-wild-type & $3(30.0)$ & $2(20.0)$ & $2(20.0)$ & $3(30.0)$ & 10 \\
\hline p53-mutant & $0(0.0)$ & $2(10.0)$ & $3(15.0)$ & $15(75.0)$ & 20 \\
\hline Total & $3(10.0)$ & 4 (13.3) & $5(16.7)$ & $18(60.0)$ & 30 \\
\hline
\end{tabular}

Abbreviation: L1CAM, L1 cell adhesion molecule.

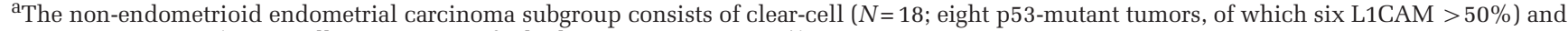
serous carcinomas $(N=12$; all p53-mutant, of which nine L1CAM $>50 \%)$.

comprising 245 cases of mixed-stage and histological subtypes, including 98 high-risk cases (Supplementary Tables 2-4). Using expression $>132.12$ as threshold for L1CAM positivity, the strongest prognostic model based on classification and regression decision tree, 64 tumors were found to be L1CAM-positive (26\%), similar to the $24 \%$ positive cases in the current high-risk series. We found that L1CAM positivity was associated with older age, advanced disease stage, non-endometrioid morphology, high tumor grade, and high-risk endometrial cancer $(P<0.001$ for all characteristics). L1CAM expression was also strongly associated with TP53 mutation in the complete study population $(P<0.001)$, in the endometrioid and nonendometrioid subtypes $(P<0.001$ and $P=0.049$, respectively), as well as in the subgroup of high-risk patients $(P<0.001)$. However, most $(75 \%)$ TP53-mutant high-risk endometrioid endometrial cancers were L1CAM-negative, confirming the results in our high-risk series.

\section{Discussion}

In this explorative study, we investigated the frequency and prognostic significance of L1CAM expression and its association with mutant p53 expression in high-risk endometrial cancers. We found that L1CAM is more frequently expressed in high-risk compared with lower-risk endometrial cancers, and is often expressed diffusely. Also, while the previously used threshold of $>10 \%$ for L1CAM positivity was not of prognostic importance in this series of high-risk endometrial cancers, a higher threshold of $>50 \%$ was associated with a higher rate of distant metastases. Finally, we showed that L1CAM positivity and mutant p53 expression are strongly associated but are not surrogate markers. 

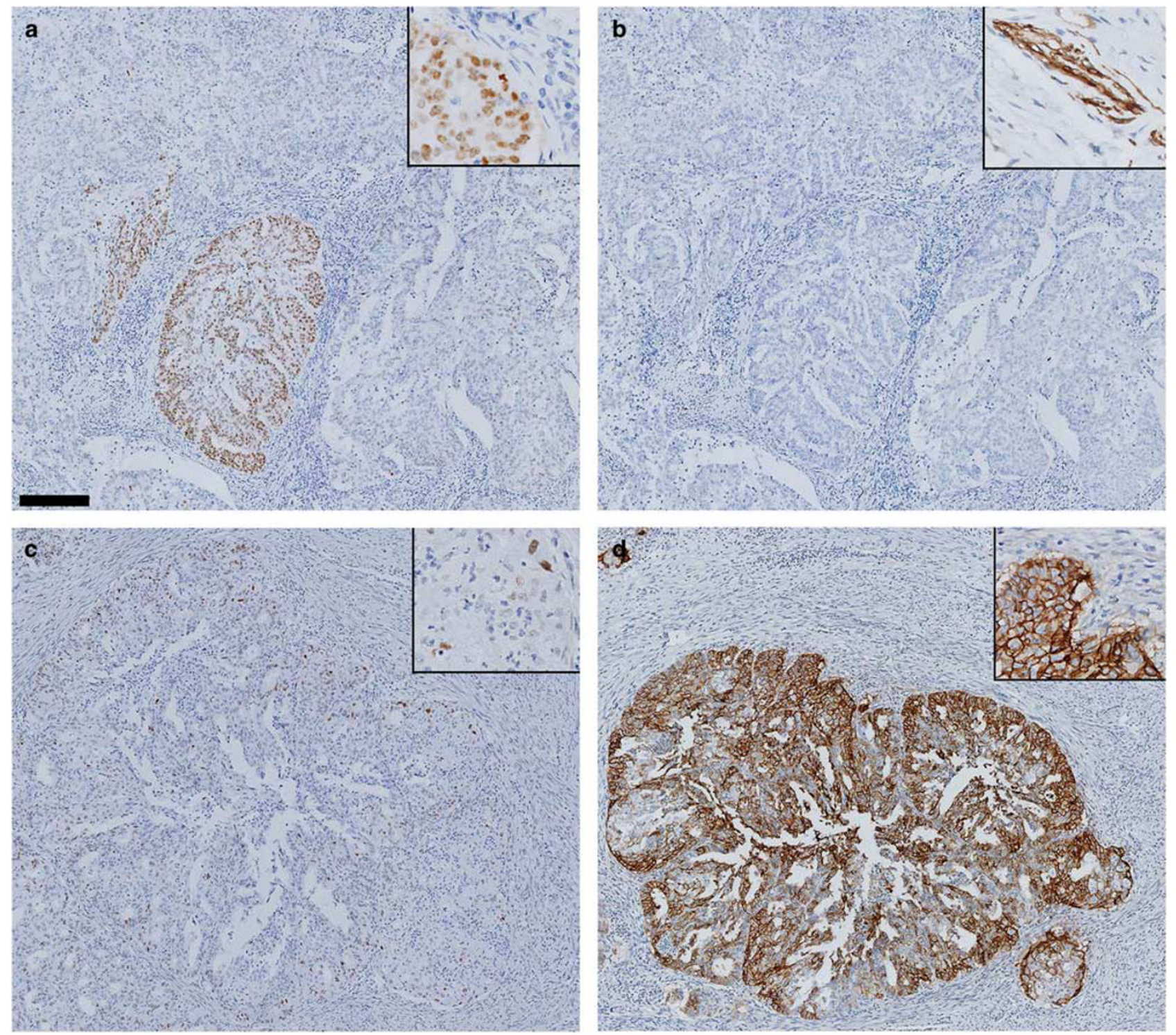

Figure 2 Immunohistochemical stainings of p53 (left column) and L1 cell adhesion molecule (L1CAM) (right column) for two cases. Scale bar represents $200 \mu \mathrm{m}$ and is the same for a-d; insets are at x40 magnification. Upper panels show a case with clonal mutant p53 expression (a), whereas the corresponding L1CAM stain is completely negative (b, nerve branch as internal positive control is shown in the inset). Lower panels provide an example of a case with wild-type p53 expression (c) but diffusely positive L1CAM expression (d).

We validated our results using the large TCGA endometrial cancers series $(N=245) .^{2}$

Using a threshold of $>10 \%$ staining for L1CAM positivity, a substantially higher rate $(44 \%)$ of L1CAM positivity was found in the high-risk series compared with the previously reported rates for lower-risk early-stage disease of $7-18 \%{ }^{10,18,19}$ Interestingly, we found a similarly increased rate when we limited our analysis to endometrioid endometrial cancers alone $(33 \%$ vs $6-17 \%),{ }^{10,18,19}$ whereas the frequency of L1CAM positivity in non-endometrioid carcinomas in our study $(77 \%)$ was similar to previous studies, such as $73 \%$ by Fogel et $a .^{10}$ These differences can largely be explained by the fact that our series contained a higher proportion of high-risk cases, with high-grade tumors and advanced disease stages.

L1CAM expression above the cutoff value of $10 \%$ for positivity was not significantly related with a higher rate of distant recurrences in this high-risk population. This is striking, because in large series of early-stage endometrial cancers, L1CAM-positive tumors (same scoring method used) demonstrated remarkably high hazard ratios-among others-for distant recurrences. ${ }^{18,19}$ Possibly because of the described considerable differences in study population, the commonly used threshold of $10 \%$ for positivity proved inappropriate for the current series of high-risk endometrial cancer. Moreover, low L1CAM expression, that is, no membranous staining 
or $<10 \%$ positivity, was unable to select patients at a low risk of recurrence in this high-risk group. Use of a higher cutoff value of $50 \%$ is supported by the previous studies: tumors with $>50 \%$ L1CAM positivity had significantly lower disease-free and overall survival rates compared with tumors with $<50 \%$ positivity $(P<0.001)$, and a higher distant recurrence rate. ${ }^{18,19}$

Contrary to endometrioid tumors, most non-endometrioid endometrial cancers $(60 \%)$ remained L1CAM-positive using the alternative cutoff value, which led us to speculate that the diffuse L1CAM expression is associated with non-endometrioid histology, and consequently with TP53 mutations. Suggestions for a relationship between L1CAM and mutant p53 expression have been made in prior endometrial cancer studies, ${ }^{18,19}$ as well as functionally in endometrial cancer cell lines. ${ }^{20} \mathrm{~A}$ link between p53 and L1CAM would also accommodate the explanation of diffuse ( $>50 \%$ ) L1CAM-positive staining in the presence of mutant p53. Such a relationship, however, would limit the value of L1CAM as an independent prognostic biomarker. A highly significant association was found between L1CAM expression and mutant p53 expression in all high-risk endometrial cancers of TransPORTEC and TCGA series, as well as in the non-endometrioid subtype. In contrast, the majority of endometrioid endometrial cancers with mutant p53 expression displayed low L1CAM expression. This indicates involvement of p53-independent activating pathways for L1CAM such as $\beta$-catenin/Wnt signaling and transforming growth factor- $\beta 1$, as may be suggested by staining at the invasive border resulting in $<50 \%$ L1CAM expression. ${ }^{24-26}$ We conclude that a strong association exists between L1CAM positivity and mutant p53 expression, but that L1CAM is not a surrogate marker of p53.

Our study is limited by its retrospective nature, modest size, limited follow-up data, and the lack of central pathology review. This study was set up as an explorative study; thus, the results should be interpreted with care. Because of these limitations, we can only suggest that L1CAM expression may have added prognostic value over TP53 mutational status, a well-known biomarker. This warrants further exploration (including a multivariate analysis) in a larger study population. Regardless of the outcome of such a study, the observation that high-risk tumors frequently display L1CAM positivity may be relevant from a therapeutic perspective, as these patients may benefit from strategies such as antibody-mediated therapy targeting L1CAM. ${ }^{27-29}$

In summary, our findings suggest that, although it has proven to be an independent prognostic factor in patients with low- and intermediate-risk features, L1CAM expression with a threshold of $>10 \%$ may not be prognostically relevant in high-risk endometrial cancer. For this group, a higher threshold of $>50 \%$ seems to be more appropriate. L1CAM expression and mutant p53 expression are strongly associated, but the two markers are not surrogates. The question remains whether L1CAM should be clinically implemented as an independent prognostic biomarker next to p53 and what threshold(s) should be used. In addition, because of the high frequency of L1CAM expression found in this study, its potential as a therapeutic target for high-risk endometrial cancer seems promising.

\section{Acknowledgments}

This work was supported by the Dutch Cancer Society (KWF-UL2012-5719; to ICvG, ES, RAN, CLC, VTHBMS, and TB). DNC was funded by a Health Foundation/Academy of Medical Sciences Clinician Scientist Fellowship. We acknowledge all clinicians, technicians, scientists, and pathologists involved in the collaborating centers within the participating groups in the TransPORTEC consortium.

\section{Disclosure/conflict of interest}

The authors declare no conflict of interest.

\section{References}

1 Nout RA, Bosse T, Creutzberg CL et al. Improved risk assessment of endometrial cancer by combined analysis of MSI, PI3K-AKT, Wnt/beta-catenin and P53 pathway activation. Gynecol Oncol 2012;126:466-473.

2 Kandoth C, Schultz N, Cherniack AD et al. Integrated genomic characterization of endometrial carcinoma. Nature 2013;497:67-73.

3 Stelloo E, Bosse T, Nout RA et al. Refining prognosis and identifying targetable pathways for high-risk endometrial cancer; a TransPORTEC initiative. Mod Pathol 2015;28:836-844.

4 Talhouk A, McConechy MK, Leung S et al. A clinically applicable molecular-based classification for endometrial cancers. Br J Cancer 2015;113:299-310.

5 Church DN, Stelloo E, Nout RA et al. Prognostic significance of POLE proofreading mutations in endometrial cancer. J Natl Cancer Inst 2015;107:402.

6 Kiefel H, Bondong S, Hazin J et al. L1CAM: a major driver for tumor cell invasion and motility. Cell Adh Migr 2012;6:374-384.

7 Rathjen FG, Schachner M. Immunocytological and biochemical characterization of a new neuronal cell surface component (L1 antigen) which is involved in cell adhesion. EMBO J 1984;3:1-10.

8 Schachner M. Neural recognition molecules and synaptic plasticity. Curr Opin Cell Biol 1997;9: 627-634.

9 Choi SY, Jo YS, Huang SM et al. L1 cell adhesion molecule as a novel independent poor prognostic factor in gallbladder carcinoma. Hum Pathol 2011;42: 1476-1483.

10 Fogel M, Gutwein P, Mechtersheimer S et al. L1 expression as a predictor of progression and survival in patients with uterine and ovarian carcinomas. Lancet 2003;362:869-875. 
11 Weinspach D, Seubert B, Schaten S et al. Role of L1 cell adhesion molecule (L1CAM) in the metastatic cascade: promotion of dissemination, colonization, and metastatic growth. Clin Exp Metast 2014;31:87-100.

12 Meier F, Busch S, Gast D et al. The adhesion molecule L1 (CD171) promotes melanoma progression. Int J Cancer 2006;119:549-555.

13 Chen DL, Zeng ZL, Yang J et al. L1cam promotes tumor progression and metastasis and is an independent unfavorable prognostic factor in gastric cancer. J Hematol Oncol 2013;6:43.

14 Kaifi JT, Reichelt U, Quaas A et al. L1 is associated with micrometastatic spread and poor outcome in colorectal cancer. Mod Pathol 2007;20:1183-1190.

15 Schroder C, Schumacher U, Fogel M et al. Expression and prognostic value of L1-CAM in breast cancer. Oncol Rep 2009;22:1109-1117.

16 Guo X, Xiong L, Zou L et al. L1 cell adhesion molecule overexpression in hepatocellular carcinoma associates with advanced tumor progression and poor patient survival. Diagn Pathol 2012;7:96.

17 Tischler V, Pfeifer M, Hausladen S et al. L1CAM protein expression is associated with poor prognosis in nonsmall cell lung cancer. Mol Cancer 2011;10:127.

18 Bosse T, Nout RA, Stelloo E et al. L1 cell adhesion molecule is a strong predictor for distant recurrence and overall survival in early stage endometrial cancer: pooled PORTEC trial results. Eur J Cancer 2014;50: 2602-2610.

19 Zeimet AG, Reimer D, Huszar $M$ et al. L1CAM in early-stage type I endometrial cancer: results of a large multicenter evaluation. J Natl Cancer Inst 2013;105: 1142-1150.

20 Schirmer U, Doberstein K, Rupp AK et al. Role of miR-34a as a suppressor of L1CAM in endometrial carcinoma. Oncotarget 2014;5:462-472.
21 Bosse T, Ter Haar NT, Seeber LM et al. Loss of ARID1A expression and its relationship with PI3K-Akt pathway alterations, TP53 and microsatellite instability in endometrial cancer. Mod Pathol 2013;26:1525-1535.

22 Lomo L, Nucci MR, Lee KR et al. Histologic and immunohistochemical decision-making in endometrial adenocarcinoma. Mod Pathol 2008;21:937-942.

23 Li B, Dewey CN. RSEM: accurate transcript quantification from RNA-Seq data with or without a reference genome. BMC Bioinform 2011;12:323.

24 Gavert N, Conacci-Sorrell M, Gast D et al. L1, a novel target of beta-catenin signaling, transforms cells and is expressed at the invasive front of colon cancers. J Cell Biol 2005;168:633-642.

25 Geismann C, Morscheck M, Koch D et al. Up-regulation of L1CAM in pancreatic duct cells is transforming growth factor beta1- and slug-dependent: role in malignant transformation of pancreatic cancer. Cancer Res 2009;69:4517-4526.

26 Huszar M, Pfeifer M, Schirmer U et al. Up-regulation of L1CAM is linked to loss of hormone receptors and E-cadherin in aggressive subtypes of endometrial carcinomas. J Pathol 2010;220:551-561.

27 Doberstein K, Harter PN, Haberkorn U et al. Antibody therapy to human L1CAM in a transgenic mouse model blocks local tumor growth but induces EMT. Int J Cancer 2015;136:E326-E339.

28 Schafer H, Dieckmann C, Korniienko O et al. Combined treatment of L1CAM antibodies and cytostatic drugs improve the therapeutic response of pancreatic and ovarian carcinoma. Cancer Lett 2012;319:66-82.

29 Arlt MJ, Novak-Hofer I, Gast D et al. Efficient inhibition of intra-peritoneal tumor growth and dissemination of human ovarian carcinoma cells in nude mice by anti-L1-cell adhesion molecule monoclonal antibody treatment. Cancer Res 2006;66:936-943.

Supplementary Information accompanies the paper on Modern Pathology website (http://www.nature.com/ modpathol) 